\title{
Effect of Inoculation with Selected Isolates of Stagonospora nodorum on Field Evaluations of Host Resistance in Winter Wheat
}

\author{
D. E. Fraser, Delta and Pine Land Company, Hartsville, SC 29550; J. P. Murphy, Department of Crop Science, \\ and S. Leath, Department of Plant Pathology, North Carolina State University, Raleigh 27695; and D. A. \\ Van Sanford, Department of Agronomy, University of Kentucky, Lexington 40546
}

\begin{abstract}
Fraser, D. E., Murphy, J. P., Leath, S., and Van Sanford, D. A. 2003. Effect of inoculation with selected isolates of Stagonospora nodorum on field evaluations of host resistance in winter wheat. Plant Dis. 87:1213-1220.

Although Stagonospora nodorum blotch occurs annually in North Carolina, selection for resistance in wheat (Triticum aestivum) breeding nurseries is hampered by the infrequent occurrence of heavy and timely disease pressure. The objective of this study was to compare estimates of host resistance in a population of 147 random winter wheat lines evaluated in epidemics produced by natural infection versus epidemics supplemented by inoculation with selected isolates. Two isolates were chosen from a set of 43 collected in North Carolina based on their aggressiveness on four wheat cultivars in a controlled environment test. Field experiments utilized a split-plot design with three replications. The main plots were inoculation treatments and the subplots were the 147 wheat genotypes. The inoculation treatments were (i) selected isolate A (more aggressive) alone, (ii) selected isolate B (less aggressive) alone, (iii) a combination of isolates A plus B, and (iv) natural infection. Selected isolate treatments were applied at Feekes growth stage 9 to 10.1 , and disease intensity was measured two or three times at 14-day intervals postinoculation. The study was conducted at one location in the 1996-97 season and two locations in the 1997-98 season. High levels of natural infection occurred, and no differences were observed among the four inoculation treatments for mean disease intensity in any of the three environments. Within environments, genotype-by-inoculation treatment variance was significant in the two environments inoculated with selected isolates at growth stage 9 but not in the environment inoculated at growth stage 10.1. Magnitudes of genetic variation and heritability for Stagonospora nodorum blotch resistance were not consistently associated with main plot treatments, and inoculation with selected isolates masked genetic variation for resistance in two treatments in one environment. Genotype rank correlations for Stagonospora nodorum blotch resistance between inoculation treatments varied from zero to 0.69 within environments, but only a single correlation between inoculation treatments in different environments was observed. Estimates of host resistance in epidemics supplemented with selected isolates did not consistently agree with estimates in epidemics produced by natural infection. Our results did not support the routine use of supplemental inoculation of wheat breeding nurseries with selected isolates of $S$. nodorum as a means of increasing genetic gain for host resistance.
\end{abstract}

Additional keywords: Septoria nodorum blotch

Stagonospora nodorum (Berk.) Castellani \& E.G. Germano (teleomorph Phaeosphaeria nodorum (E. Müller) Hedjaroude), the causal organism of Stagonospora nodorum blotch (SNB), is found in most temperate climates where wheat (Triticum aestivum L.) is grown (23). Extensive information has been gathered on

Corresponding author: D. E. Fraser

E-mail: dawn.e.fraser@deltaandpine.com

This research was supported in part by the North Carolina Agricultural Research Service, North Carolina State University, Raleigh, and by a grant from the North Carolina Small Grain Growers Association, Inc.

Accepted for publication 16 May 2003.

Publication no. D-2003-0723-01R

(C) 2003 The American Phytopathological Society epidemiology, host-pathogen interactions, pathogen biology, and host resistance, but progress in developing cultivars with high levels of resistance in the eastern United States has been modest (47). Confounding factors that hamper breeding for host resistance include a lack of continuous selection pressure, low heritability, and sporadic epidemics (10).

Studies investigating the genetics of resistance to SNB have reported polygenic control with both dominant and recessive additive gene effects (4-6,9,20,32$34,37,41,50)$. Studies investigating the pathogenicity of $S$. nodorum populations have found high levels of variation for aggressiveness, expressed as differences among isolates in their relative abilities to cause disease on a host that contains only race nonspecific resistance to pathogenesis $(1,19,38,44)$. Recent research in the United States and Europe suggested that large geographical regions contain a single genetic population of the fungus $(16,17,27)$. These studies reported a high level of genetic diversity and low levels of clonality within the fungal population.

A large number of studies have measured a spectrum of host resistance components under diverse environmental conditions $(13,18,21,39)$. Inoculation with mixtures of fungal isolates was common $(3,4,9,15,37,50)$, but in several studies inoculations using single isolates have been made $(8,14,33,49)$. Significant isolate-by-cultivar interaction has been reported $(1,2,19,42,45)$, but the magnitude was small relative to cultivar and isolate sources of variation. In contrast, a few studies $(12,31,43)$ reported the absence of significant cultivar-by-isolate interaction. Although some level of gene-for-gene interaction has been demonstrated in controlled studies with selected host genotypes inoculated with selected isolates, SNB resistance is generally considered nonspecific under field conditions where populations of the pathogen confront populations of the host (40). There are differences in opinion on the use of single versus multiple isolates in host screening. Arseniuk and Czembor (2) concluded that a single, selected, aggressive isolate might be more reliable than a mixture of isolates in screening for SNB resistance. McDonald et al. (28) and Mebrate and Cooke (31) regarded screening with isolate mixtures to be of paramount importance. Although little evidence of widely dispersed clones exists, it can be argued that a representative sample of the diversity in virulence in a population requires a mixture of isolates (28).

It is common practice for breeders to rely on epidemics produced by naturally occurring $S$. nodorum populations for selection of SNB-resistant genotypes in their nurseries (35). In North Carolina, where SNB occurs annually but reaches epidemic proportions with a frequency of less than one year in two, the routine inoculation of breeding nurseries with isolate spore suspensions prior to head emergence might improve selection efficiency. Cunfer et al. (7) used a combination of noninoculated and inoculated nurseries to develop four SNB-resistant germ plasm lines in Georgia. Accurate long-term prediction of the environmental variables that promote natural disease development is not possible, 
thus inoculation would be necessary every season to ensure timely disease development. It is unlikely that a single introduced isolate would be the only, or perhaps even the predominant, isolate in any given season. Thus, it is timely to examine the use of selected isolates in a breeding nursery where naturally occurring $S$. nodorum populations are present, and wheat genotypes ranging from susceptible to moderately resistant are being evaluated. The objective of this study was to compare estimates of host resistance to SNB in a population of soft red winter wheat genotypes evaluated in epidemics produced by natural inoculum alone versus epidemics supplemented by inoculation with selected isolates.

\section{MATERIALS AND METHODS}

Collection and screening of isolates of $S$. nodorum. Forty-three leaf samples exhibiting symptoms of infection by $S$. nodorum were collected from wheat fields in Washington, Lenoir, Wayne, Johnston, and Rowan counties, North Carolina, in June 1996. After surface disinfecting leaves in $95 \%$ ethanol for $10 \mathrm{~s}$ then in $10 \%$ commercial bleach solution for $30 \mathrm{~s}$ and rinsing them for $1 \mathrm{~min}$ in sterile distilled water, a single lesion per leaf was excised and transferred to a petri plate containing potato dextrose agar (PDA; $20 \mathrm{~g}$ of PDA and $7 \mathrm{~g}$ of agar in 1 liter of $\mathrm{H}_{2} \mathrm{O}$ ). The plates were placed in a growth chamber for 3 to 7 days at a constant $20^{\circ} \mathrm{C}$ with alternating $12 \mathrm{~h}$ of light and darkness. Fungal hyphal tips were removed from the edges of colonies and transferred to fresh plates of PDA. Colonies that developed after 3 to 5 days were confirmed as $S$. nodorum by microscopic examination of pycnidiospores.

Seeds of wheat cultivars Saluda (PI 480474), Caldwell (CItr 17897), Coker 9733 (PI 601428), and Coker 9766 (PI 601429) were planted in 12-cm-diameter polystyrene cups containing MetroMix (Scotts-Sierra Horticultural Products Co., Marysville, $\mathrm{OH}$ ), soil, and sand in a 4:2:1 ratio. A liquid fertilizer with the appropriate balance of major and minor nutrients was applied. These four cultivars represented diversity in geographical origin and pedigrees within the soft red winter wheat germ plasm pool. Cups were placed in a growth chamber in the North Carolina State University Phytotron with day/night temperatures of $22 / 18^{\circ} \mathrm{C}$ and alternating 12 $\mathrm{h}$ light and darkness until plants reached the three- to four-leaf stage. The experimental design was a split plot with two replications where isolate was the main plot factor and cultivar was the subplot factor. An experimental unit consisted of a single plant of one cultivar growing in a single cup.

Fungal hyphal tips were removed from the edges of PDA plates containing each of the 43 isolates and placed on fresh plates of PDA prepared as described above. Cultures were grown for 7 days under the same temperature and light regimes as described previously, and inoculum solutions were prepared by adding sterile distilled water to 7-day-old plates of each of the 43 isolates. Cirrhi containing pycnidiospores were dislodged with a sterile plastic plate scraper. Each isolate suspension was adjusted to $1 \times 10^{6}$ spores $\mathrm{ml}^{-1}$ based on spore counts in a hemacytometer, and a drop of Tween 20 was added. A hand-held atomizer was utilized to apply each spore suspension to the four tester cultivars until runoff occurred inside a laminar flow hood. A solution containing only distilled water and Tween 20 was applied to a set of control plants. Prior to inoculation, the base of the third leaf of each plant was marked with a nonphytotoxic felt tipped pen, and the leaf area was calculated by measuring the length and width of the marked leaves.

The plants were returned to the growth chamber after inoculation and maintained at 12 -h day/night temperatures of $22 / 18^{\circ} \mathrm{C}$. Relative humidity was maintained at approximately $100 \%$ by an automatic system that applied $15 \mathrm{~s}$ of water mist every 15 min. Marked leaves were checked daily, and the date of initial symptom appearance was recorded. Ten days after the initial symptoms appeared, the total number of lesions and the percentage of diseased tissue on each of the marked leaves were recorded. Lesion number data underwent square root transformation, and percentage of diseased tissue data underwent arcsine transformation prior to analysis of variance with the GLM procedure of the SAS software package (SAS Institute, Inc., Cary, $\mathrm{NC}$ ). Isolates and cultivars were considered random variables, and the significance of main, subplot, and interaction effects were tested using the RANDOM and TEST statements. Variance components were estimated by equating actual to expected mean squares. Means for all significant effects were separated using the Duncan's multiple range test with alpha $=0.05$. The two isolates selected (isolates $\mathrm{A}$ and B) had contrasting mean values for percentage of leaf area diseased $(P<0.01)$.

Host population. The host population contained 147 genotypes representing a random sample of the germ plasm found in wheat cultivar development nurseries in the southeastern United States. These included 125 random $\mathrm{F}_{3: 5}, \mathrm{~F}_{4: 6}$, and $\mathrm{F}_{5: 7}$ lines from the North Carolina State University wheat cultivar development nursery grown in the 1995-96 season. The nursery contained over 10,000 breeding lines, and each of the 125 randomly selected lines came from a different cross. Seeds of these lines were harvested at the Cunningham Research and Education Center, Kinston, NC, in June 1996. Nineteen advanced generation lines were also randomly chosen from the 1995-96 Uniform Southern Soft Red
Winter Wheat Yield Nursery harvested at the same location. These lines came from four public and three private breeding programs. The cultivars Caldwell, Coker 9733, and Coker 9766 used in the isolate screening were included three times per replication. Saluda, also utilized in isolate screening, was not included in field evaluations due to its extreme susceptibility to powdery mildew (caused by Blumeria graminis (DC.) E.O. Spear f. sp. tritici Em. Marchal). The host population comprising these 147 lines was evaluated in the 199697 and the 1997-98 seasons, as described below.

Untreated seed harvested in June 1996 was used for planting in both evaluation seasons. Laboratory tests to detect the presence of $S$. nodorum on seeds were conducted on a random sample of 20 genotypes using the method of Manandhar and Cunfer (26). One hundred seeds per genotype were placed in petri dishes containing oxgall agar and then placed in a darkened chamber at room temperature for 5 days. Presence of $S$. nodorum was detected if developing colonies fluoresced when illuminated with black light.

Field evaluations. During the 1996-97 season, the 147 genotypes were evaluated for resistance to SNB at the Upper Mountain Research Station, Laurel Springs, NC. During the 1997-98 season, the genotypes were evaluated for resistance to SNB at Laurel Springs and Kinston. Planting date at Laurel Springs (elevation $875 \mathrm{~m}$ ) was 23 September in both 1996 and 1997. Planting date at Kinston (elevation $18 \mathrm{~m}$ ) was 21 October 1997.

The experimental design was a spilt-plot with three replications in all environments. The main plot factor was inoculation treatment and the subplots were the 147 genotypes. Inoculation treatments were (i) inoculation with isolate $\mathrm{A}$, (ii) inoculation with isolate $\mathrm{B}$, (iii) inoculation with a mixture of isolates $\mathrm{A}$ and $\mathrm{B}$ in equal proportions, and (iv) natural infection in which disease developed from the naturally occurring background inocula. Twenty seeds of each genotype were planted together in hill plots on a $0.3-\mathrm{m}$ grid square pattern. A main plot consisted of groups of 147 genotypes planted in hill plots, surrounded on all sides by a $1.75-\mathrm{m}$ border of the triticale ( $\times$ Triticosecale Wittmack) cultivar Resource Seeds WI X53-238. When approximately $50 \%$ of the genotypes in a population reached Feekes GS 10.1 (22) in 1997 and GS 9 in 1998, inocula for the three treatments were prepared as described previously, calibrated to $1 \times 10^{6}$ spores per $\mathrm{ml}$, and applied over the top in approximately 12 liters of water using a backpack sprayer with a 3-m boom. Care was taken to avoid cross contamination among treatments during the inoculation process by triple-rinsing the spray tank and changing spray suits between treatments. At the time of inoculation, the triticale 
border was approximately $0.5 \mathrm{~m}$ taller than the wheat genotypes under test, and inoculations were conducted late in the day to avoid any spore dispersal by wind.

Foliar and head disease intensity was recorded on each genotype using the SaariPrescott double-digit scale (00 to 99) (11). The first digit indicated the relative height of infection $(5=$ midplant and $9=$ head $)$, and the second digit indicated the severity of disease on the highest infected leaf or the head on a scale of 0 to 9 . Ratings were taken at 2, 4, and 6 weeks postinoculation at Laurel Springs 1997 and Kinston. A storm between the second and third ratings at Laurel Springs 1998 lodged $90 \%$ of the plots; thus ratings were not taken 6 weeks postinoculation in this environment. Host resistance was estimated as the mean of the three ratings in Laurel Springs 1997 and Kinston, and the mean of the two ratings at Laurel Springs 1998. Days to heading (day on which $50 \%$ of the heads in a plot had fully emerged from the boot) and plant height were recorded on three noninoculated replications of each genotype at Kinston. Plant height was measured as the height from the base of the plant to the tip of the heads, excluding awns, taken 14 days after heads were fully emerged.

Disease intensity, heading date, and plant height data were analyzed by analysis of variance with the GLM procedure of the SAS software. Mean disease intensity for each genotype in each of the 12 inoculation treatment-environment combinations was regressed on mean genotype heading date and plant height to assess the impact of these variables on host resistance estimates (48). None of the regressions were significant at the 0.05 level; therefore, the disease intensity ratings were not adjusted for genotype maturity or stature. Inoculation treatments were considered fixed, and genotypes and environments were considered random effects in the analysis of disease intensity. The mixed model outlined by McIntosh (30) was followed. Significances of main, subplot, and interaction effects were tested using the RANDOM and TEST statements. Spearman rank correlation coefficients between genotypic means in the various inoculation treatments were calculated using the CORR procedure. Variance components for random effects and their standard errors were estimated using the method of restricted maximum likelihood in the MIXED procedure with the COVTEST statement (24). Variance components were utilized to estimate entry mean heritabilities of the host within each of the three environments according to Nyquist (36). Finally, in order to graphically represent the relationships between the 12 inoculation treatmentenvironment combinations, a 12 by 147 matrix of genotype means was subjected to GGE Biplot analysis (51). In this analysis, the first two principal components (PC1 and $\mathrm{PC} 2$ ) of a singular value decomposi- tion of the genotype-by-environment effects matrix were used to approximate the original data matrix. The 12 inoculation treatment-environment combinations were displayed graphically in a two-dimensional biplot, the $x$ and $y$ axes of which are PC1 and PC2, respectively. A polygon was drawn joining the genotypes furthest from the biplot origin. Lines drawn from the biplot origin perpendicular to the polygon sides defined mega-environments. Megaenvironments were relatively homogeneous subsegments of all the environments, and each had a different top-ranking genotype.

\section{RESULTS AND DISCUSSION}

Screening of isolates of $S$. nodorum. Significant variation was observed among the 43 isolates of $S$. nodorum for percentage of leaf area diseased, but not for lesions per square centimeter (Table 1). Significant variation was observed among both cultivar and cultivar-by-isolate sources of variation for both disease components. Thirty-two of the 43 isolates produced lesions on all four test cultivars, and nine isolates produced lesions on three cultivars. Single isolates produced up to $80 \%$ diseased tissue on Coker 9733, 65\% on Coker 9766, 40\% on Caldwell, and 35\% on Saluda. A different isolate was the most aggressive on each of the four cultivars. The 43 isolates produced 14 different ranking patterns among the four cultivars. The most common pattern, with rankings similar to that observed for the 43 isolates overall (Table 2 ), was produced by seven isolates. Saluda was the most resistant for both disease components averaged over all 43 isolates, and Coker 9766 was the most susceptible.

These results were similar to those reported by Allingham and Jackson (1) using 282 isolates collected in northern Florida, and by other researchers working with fewer isolates $(12,19,38,43,44)$. The cultivar source of variation was almost threefold the isolate source of variation for percentage of leaf area diseased. Similar relative differences were reported in other studies $(2,43)$ and underscored the greater role of genotypic differences among cultivars relative to genotypic differences among isolates in determining disease reactions. Based on the relative amounts of disease they caused, there was no geographical clustering of aggressive isolates, which was in agreement with other studies $(1,42)$.

In contrast to studies that reported small or no significant cultivar-by-isolate interactions $(2,31,38,42,43)$, we observed an interaction term for percentage of leaf area diseased that was approximately twofold the cultivar variance component. Cultivars differed in their disease reactions depending on the isolate used, which indicated that judicious choice of isolate is important if a screening program is based on the use of a single isolate compared with screening using a bulk of isolates, each differing in the level of aggressiveness. The cultivarby-isolate term for lesions per square centimeter was one-half the magnitude of the cultivar component and, thus, more in line with reports in previous studies $(2,31,38)$.

Table 1. Analyses of variance of arcsine percentage of leaf area diseased and square root lesions per square centimeter recorded on four wheat cultivars inoculated with 43 isolates of Stagonospora nodorum

\begin{tabular}{lccc}
\hline & & \multicolumn{2}{c}{ Mean squares $^{\mathbf{z}}$} \\
\cline { 4 - 4 } Source of variation & $\mathbf{d f}$ & Leaf area diseased (\%) $^{*}$ Lesions/cm $^{\mathbf{2}}$ \\
\hline Replication (R) & 1 & $0.05^{*}$ & 0.73 \\
Isolate (I) & 42 & $0.12^{*}$ & 1.09 \\
R $\times$ I & 42 & 0.01 & 0.69 \\
Cultivar (C) & 3 & $1.44^{* *}$ & $26.57^{* *}$ \\
C $\times$ I & 126 & $0.07^{* *}$ & $0.99^{* *}$ \\
Error & 129 & 0.008 & 0.65 \\
CV \% & & 23.7 & 47.7 \\
Mean & & 18.0 & 3.4 \\
Range & $7.6-39.0$ & $1.3-11.8$ \\
\hline
\end{tabular}

$\mathrm{z} *$ and $* *$ indicate significance at 0.05 and 0.01 probability levels, respectively.

Table 2. Percentage of leaf area diseased and lesions per square centimeter observed on four wheat cultivars inoculated with 43 isolates of Stagonospora nodorum and corresponding means for the selected isolates A and B utilized in subsequent field inoculation

\begin{tabular}{|c|c|c|c|c|c|c|}
\hline \multirow[b]{2}{*}{ Cultivar } & \multicolumn{2}{|c|}{ All 43 isolates } & \multicolumn{2}{|c|}{ Isolate A } & \multicolumn{2}{|c|}{ Isolate B } \\
\hline & $\begin{array}{c}\text { Leaf area } \\
\text { diseased }(\%)\end{array}$ & $\begin{array}{c}\text { Lesions/ } \\
\text { cm }^{2}\end{array}$ & $\begin{array}{c}\text { Leaf area } \\
\text { diseased }(\%)\end{array}$ & $\begin{array}{c}\text { Lesions/ } \\
\text { cm }^{2}\end{array}$ & $\begin{array}{c}\text { Leaf area } \\
\text { diseased }(\%)\end{array}$ & $\begin{array}{c}\text { Lesions/ } \\
\text { cm }^{2}\end{array}$ \\
\hline Saluda & $13.88 \mathrm{a}^{\mathrm{z}}$ & $1.34 \mathrm{a}$ & $35.45 \mathrm{a}$ & $0.94 \mathrm{a}$ & $25.47 \mathrm{a}$ & $1.49 \mathrm{a}$ \\
\hline Caldwell & $21.10 \mathrm{~b}$ & $2.72 \mathrm{~b}$ & $15.66 \mathrm{a}$ & $1.96 \mathrm{a}$ & $4.01 \mathrm{a}$ & $1.30 \mathrm{a}$ \\
\hline Coker 9733 & $22.33 \mathrm{~b}$ & $2.25 \mathrm{~b}$ & $39.05 \mathrm{a}$ & $9.67 \mathrm{~b}$ & $9.21 \mathrm{a}$ & $0.94 \mathrm{a}$ \\
\hline Coker 9766 & $33.36 \mathrm{c}$ & $6.10 c$ & $59.32 \mathrm{~b}$ & $2.79 \mathrm{a}$ & $22.95 \mathrm{a}$ & $2.99 \mathrm{a}$ \\
\hline
\end{tabular}

${ }^{\mathrm{z}}$ Means within a column followed by the same letter are not significantly different according to Duncan's multiple range test $(P \leq 0.05)$. 
Two isolates with significant differences in percentage of leaf area diseased were selected for use in field inoculations. Isolate $\mathrm{A}$ had a mean percent leaf area diseased value of 32.7 , and isolate $B$ had a mean value of 9.6. Both isolates were collected in Washington County, NC. The more aggressive isolate A elicited significant differences among the four test cultivars for both disease components, but the less aggressive isolate $\mathrm{B}$ did not elicit significant differences among the four cultivars for either disease component (Table 2).

Field evaluations of the 147 genotypes. Environmental factors in both seasons were favorable for SNB epidemics in the field nurseries (Table 3). High levels of natural inoculum were present in all environments as indicated by mean disease intensity values in the natural infection treatment. Coefficients of variation and determination and overall entry mean heritability estimates within each environment indicated that the experimental protocol and disease rating technique provided conditions that allowed the effects of the treatments to be observed and rated satisfactorily. Entry mean heritability estimates indicated that Laurel Springs 1997 was most favorable for making genetic progress in disease resistance and Laurel Springs 1998 was the least favorable. Interaction of subplot effects with environments was highly significant.

Significant differences were not observed among the four inoculation treatments for mean disease intensity in any of the three environments (Table 3). For example, no treatment resulted in consistently higher or lower disease intensities, and the impact of inoculation with selected isolates, either singly or in combination, did not result in mean disease intensities that were different from the natural infec- tion treatment. The different estimates of isolate aggressiveness we obtained in controlled environment tests were not reflected in mean disease levels in field nurseries.

Laboratory tests to detect $S$. nodorum in seed of the genotypes planted in our field studies indicated a range of 5 to $7 \%$ infection. Seed infection levels reported as sufficient to initiate an epidemic range from less than $1 \%(12,46)$ to $10 \%(25)$. The level of seed infection found in the subsample of genotypes tested in this study was sufficient to initiate epidemics in the noninoculated treatments, but not at a level to eliminate the effects of other isolates naturally migrating into plots or the effects of the applied isolate treatments.

Significant differences were observed among host genotypes when analyzed across the four inoculation treatments within each environment (Table 3). Genotype-by-inoculation treatment variance was significant in Laurel Springs 1998 and Kinston, but not in Laurel Springs 1997. These results were reflected in the Spearman rank correlation coefficients between the means of the 147 genotypes evaluated in the four inoculation treatments (Table 4). All correlations among inoculation treatments were significantly positive at Laurel Springs 1997, and the mean correlation coefficient was 0.65 . The results for Laurel Springs 1998 were in sharp contrast, in that only one of the six correlations was significant. Results from Kinston 1998 were intermediate between Laurel Springs 1997 and Laurel Springs 1998 with respect to rank correlations among the four inoculation treatments. Although four of the six correlation coefficients were significant, the mean correlation was only 0.25 .

Timing of inoculation in 1997 (Feekes GS 10.1) versus 1998 (Feekes GS 9) may have contributed to the differences in num- ber and magnitudes of the significantly positive correlations in Laurel Springs 1997 versus the other two environments. The significant correlations among inoculation treatments at Laurel Springs 1997 suggested that an epidemic had developed in all treatments based on natural inoculum present at this location, which was little influenced with respect to genotype rankings by supplemental inoculation with selected isolates.

The mean disease intensity and genetic variance in the natural infection treatment in Laurel Springs 1998 indicated that an epidemic developed from background inoculum that resulted in the expression of a high level of genetic variation for host resistance (Table 3 ). In contrast, there were no significant variations among genotypes in the treatments inoculated with isolate A alone and isolate $\mathrm{B}$ alone. These results contributed to the large genotype-byinoculation treatment interaction, plus the relatively small genotypic variance over all inoculation treatments at this location. The entry mean heritability was only $27 \%$ for the combination treatment with both isolates A plus B in comparison to $60 \%$ for the natural infection treatment, and zero for either the isolate A or isolate B treatments.

Results at Laurel Springs 1998 may reflect the impact of the selected isolates on expression of host resistance, the method of inoculation with the selected isolates, the subsequent disease development, or the absence of a third disease intensity rating. Environmental constraints to disease development were not obvious, as precipitation was evenly distributed during the postinoculation period and averaged 2.5 $\mathrm{cm}$ per week. Temperatures were in the optimum range for infection (mean daily high temperature of $27.2^{\circ} \mathrm{C}$ and mean daily low temperature of $12.2^{\circ} \mathrm{C}$ ). The residual variances for the treatments inoculated

Table 3. Mean disease intensity, variance components due to genotypes and genotype $\times$ inoculation treatment interactions, entry mean heritabilities, and coefficients of variation and determination based on 147 wheat genotypes evaluated in four inoculation treatments with Stagonospora nodorum in three environments in the 1997 and 1998 seasons

\begin{tabular}{|c|c|c|c|c|c|c|c|c|c|}
\hline \multirow[b]{2}{*}{$\begin{array}{l}\text { Inoculation } \\
\text { Treatment }\end{array}$} & \multicolumn{3}{|c|}{ Laurel Springs (1997) } & \multicolumn{3}{|c|}{ Laurel Springs (1998) } & \multicolumn{3}{|c|}{ Kinston (1998) } \\
\hline & $\begin{array}{c}\text { Disease } \\
\text { intensity }^{\mathrm{w}}\end{array}$ & $\sigma_{G}^{2}{ }^{x}$ & $\begin{array}{c}\text { Heritability } \\
(\%)\end{array}$ & $\begin{array}{c}\text { Disease } \\
\text { intensity }\end{array}$ & $\sigma_{G}^{2}$ & $\begin{array}{c}\text { Heritability } \\
(\%)\end{array}$ & $\begin{array}{c}\text { Disease } \\
\text { intensity }\end{array}$ & $\sigma_{G}^{2}$ & $\begin{array}{c}\text { Heritability } \\
(\%)\end{array}$ \\
\hline $\begin{array}{l}\text { Isolate A } \\
\text { Range }\end{array}$ & $\begin{array}{c}80 \\
65-89\end{array}$ & $14.0 \pm 2.4$ & 64 & $\begin{array}{c}55 \\
45-73\end{array}$ & ns & 0 & $\begin{array}{c}48 \\
38-63\end{array}$ & $10.7 \pm 2.5$ & 44 \\
\hline Range & $65-89$ & & & $45-73$ & & & $38-63$ & & \\
\hline Isolate B & 81 & $13.0 \pm 2.2$ & 62 & 47 & $\mathrm{~ns}$ & 0 & 48 & $13.5 \pm 2.7$ & 52 \\
\hline Range & $69-90$ & & & $32-64$ & & & $33-62$ & & \\
\hline Isolate A-B & 81 & $15.7 \pm 2.9$ & 55 & 52 & $13.2 \pm 4.8$ & 27 & 50 & $16.7 \pm 3.2$ & 45 \\
\hline Range & $65-89$ & & & $37-69$ & & & $37-63$ & & \\
\hline Natural infection & 79 & $12.6 \pm 2.4$ & 54 & 51 & $42.8 \pm 7.5$ & 60 & 45 & $15.8 \pm 3.2$ & 50 \\
\hline Range & $65-89$ & & & $30-71$ & & & $32-60$ & & \\
\hline $\mathrm{CV} \%$ & 5.6 & & & 18.2 & & & 11.2 & & \\
\hline$R^{2 \mathrm{y}}$ & 0.64 & & & 0.59 & & & 0.68 & & \\
\hline Heritability (\%) & 88 & & & 25 & & & 62 & & \\
\hline$\sigma_{\mathrm{G}}^{2}$ & $13.4 \pm 1.8$ & & & $3.0 \pm 1.5$ & & & $7.0 \pm 1.4$ & & \\
\hline$\sigma_{\mathrm{G} \times \mathrm{TT}^{\mathrm{z}}}^{2}$ & ns & & & $7.1 \pm 2.7$ & & & $7.2 \pm 1.2$ & & \\
\hline
\end{tabular}

${ }^{\mathrm{w}}$ Disease intensity was recorded using the Saari-Prescott double-digit scale $(00$ to 99$)$ where the first digit indicated the relative height of infection $(5=$ midplant and $9=$ head) and the second digit indicated the severity of disease on the highest infected leaf or the head on a scale of 0 to 9.

${ }^{x}$ Genotypic variance.

y Coefficient of determination.

${ }^{\mathrm{z}}$ Genotype $\times$ inoculation treatment interaction variance. 
with isolate $\mathrm{A}$ alone and isolate $\mathrm{B}$ alone were 57 to $104 \%$ greater than the residual variance in the natural infection treatment. This indicated disease development was uneven across replications over the 4 weeks following inoculation and suggested that the uneven disease development was the major contributor to the results observed. The absence of a third disease intensity rating was probably not a contributing factor because analyses based on two ratings only at the other locations resulted in the same overall results and conclusions as when based on data from three ratings (data not shown).

Significant genetic variation was observed within each of the four inoculation treatments at Kinston (Table 3). The magnitudes of the variance components were similar to those observed in Laurel Springs 1997 with the exception of the isolate A treatment. Heritability of SNB resistance was similar within each of the four treatments. Mean disease intensity values for Kinston in 1998 indicated that disease development in treatments inoculated with selected isolates was more uniform across replications than in Laurel Springs 1998. Host response in plots at Kinston in 1998 was influenced to a greater degree by inoculation with selected isolates than in Laurel Springs 1997, where the natural background inoculum had the greatest influence on the level of disease development in all treatments.

Approximately two-thirds of the rank correlations between genotypes evaluated in different inoculation treatments were significantly positive within environments; yet there was almost complete absence of significant rank correlations between environments (Table 4). In the combined analysis across environments, the inoculation treatment-by-environment variance was not significant, and the genotype-byenvironment variance component was approximately twice the genotype-byenvironment-by-inoculation treatment vari- ance component. Because large geographical regions contain a single genetic population of $S$. nodorum $(16,27)$, it is unlikely that variation in the natural inoculum was a major contributor to genotype-by-environment interaction. The importance of evaluation of genotypes in multiple environments is clear, and the influence of the environment on disease expression in both inoculated and natural infection treatments is illustrated by the lack of significant rank correlations between environments.

The GGE biplot analysis (Fig. 1) illustrates the primary influence of environment rather than inoculation treatment on disease resistance estimates. The four inoculation treatments at Laurel Springs 1997 and Kinston formed two tight, but distinct, clusters. The relative dispersion of the Laurel Springs 1998 inoculation treatments reflected the masking of genetic variation in treatments inoculated with isolate A or isolate $B$ versus the significant variation observed in the treatment with a combination of isolates and natural infection. For example, inoculation with isolate $\mathrm{A}$ alone and isolate $\mathrm{B}$ alone revealed no variation among the 147 genotypes. These treatments lay close to the biplot origin and were ineffective in distinguishing among genotypes. The four treatments at Laurel Springs 1997 were more discriminating among genotypes than the Kinston treatments as evidenced by their relative distances from the biplot origin. It was notable that none of the treatments inoculated

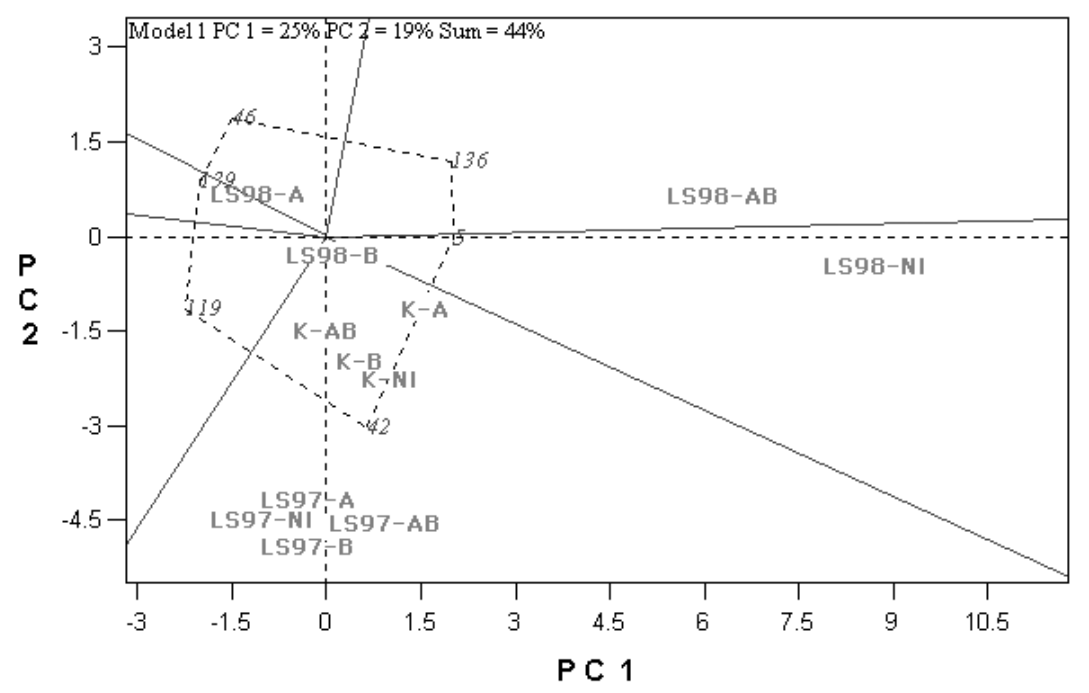

Fig. 1. Polygon view of GGE biplot of 147 wheat genotypes evaluated under 12 inoculation treatment-environment combinations. Inoculation treatments are denoted as isolate A (A), isolate B (B), isolates A and B combined (AB), and natural infection (NI). Environments are denoted as Kinston (K), Laurel Springs 1997 (LS97), Laurel Springs 1998 (LS98). Entry numbers at the vertices of the polygon represent entries furthest from the biplot origin. The two principal components (PC1 and PC2) are linear combinations of the original variables that explain differing amounts of variation in the original data set. Given $\mathrm{i}$ genotypes and $\mathrm{j}$ locations, the $\mathrm{i} \times \mathrm{j}$ two-way table of genotype/locations means can be broken down through singular value decomposition in $\mathrm{k}$ principal components, where $\mathrm{k}$ is the lesser of $\mathrm{i}$ or $\mathrm{j}$.

Table 4. Spearman rank correlation coefficients between disease intensity measurement on 147 wheat genotypes evaluated under four inoculation treatments with Stagonospora nodorum in three environments in North Carolina in 1997 and 1998

\begin{tabular}{|c|c|c|c|c|c|c|c|c|c|c|c|c|}
\hline \multirow[b]{2}{*}{ Treatment } & \multicolumn{4}{|c|}{ Laurel Springs (1997) } & \multicolumn{4}{|c|}{ Laurel Springs (1998) } & \multicolumn{4}{|c|}{ Kinston (1998) } \\
\hline & $\mathbf{A}$ & B & $\mathbf{A}+\mathbf{B}$ & NI & $\mathbf{A}$ & B & $\mathbf{A}+\mathbf{B}$ & NI & $\mathbf{A}$ & B & $\mathbf{A}+\mathbf{B}$ & NI \\
\hline \multicolumn{13}{|c|}{ Laurel Springs $(1997)^{\mathrm{z}}$} \\
\hline A & $\ldots$ & 0.64 & 0.60 & 0.64 & ns & ns & ns & ns & ns & ns & ns & ns \\
\hline B & & $\ldots$ & 0.69 & 0.67 & ns & $\mathrm{ns}$ & $\mathrm{ns}$ & $\mathrm{ns}$ & ns & $\mathrm{ns}$ & $\mathrm{ns}$ & ns \\
\hline$A+B$ & & & $\ldots$ & 0.67 & ns & ns & ns & $\mathrm{ns}$ & ns & $\mathrm{ns}$ & $\mathrm{ns}$ & $\mathrm{ns}$ \\
\hline NI & & & & $\ldots$ & ns & ns & $\mathrm{ns}$ & ns & ns & ns & ns & $\mathrm{ns}$ \\
\hline \multicolumn{13}{|c|}{ Laurel Springs (1998) } \\
\hline A & & & & & $\ldots$ & ns & ns & ns & ns & ns & $\mathrm{ns}$ & ns \\
\hline B & & & & & & $\ldots$ & ns & $\mathrm{ns}$ & ns & $\mathrm{ns}$ & $\mathrm{ns}$ & $\mathrm{ns}$ \\
\hline $\mathrm{A}+\mathrm{B}$ & & & & & & & $\ldots$ & 0.59 & 0.21 & ns & ns & $\mathrm{ns}$ \\
\hline NI & & & & & & & & $\ldots$ & ns & ns & ns & ns \\
\hline \multicolumn{13}{|c|}{ Kinston (1998) } \\
\hline A & & & & & & & & & $\ldots$ & 0.27 & 0.24 & 0.43 \\
\hline B & & & & & & & & & & $\ldots$ & 0.56 & ns \\
\hline$A+B$ & & & & & & & & & & & $\ldots$ & $\mathrm{ns}$ \\
\hline NI & & & & & & & & & & & & $\ldots$ \\
\hline
\end{tabular}

${ }^{\mathrm{z}}$ All numeric values significant at the 0.01 probability level. 
with selected isolates exhibited a greater ability to discriminate among genotypes than did the natural infection treatments.

Nine of the 12 inoculation treatmentenvironment combinations fell into one mega-environment. The remaining inoculation treatment-environment combinations (Laurel Springs 1998 natural infection, Laurel Springs 1998 combination treatment A and B, Laurel Springs 1998 treatment A), fell into three distinct megaenvironments. This reflected the overall lack of genotype rank correlations among treatments in this environment. The pedigree of the most resistant genotype (entry 42) in the mega-environment containing the majority of inoculation treatmentenvironment combinations was one-half Coker 9733, the moderately resistant control. Entries 5 and 136, which contained $100 \%$ southeastern U.S. adapted germ plasm, were the resistant genotypes in the mega-environments containing Laurel Springs 1998 natural infection and the combination of isolate A and B treatments, respectively. In contrast, in the same megaenvironments, entries 46, 139, and 119 had poor levels of resistance overall and contained 12.5 to $50 \%$ exotic germ plasm in their pedigrees.

Selection of resistant genotypes. In the natural infection treatment at Laurel Springs 1998 and Kinston, the two moderately resistant cultivars, Coker 9733 and Caldwell, had significantly less disease than the susceptible control Coker 9766 (Table 5). No significant differences in disease intensity were observed among these cultivars at Laurel Springs 1997 in the natural infection treatment. In comparisons between control cultivar disease ratings based on selected isolate and natural infection treatments, the greatest correspondence was observed between the isolate A treatment and the natural infection treatment. In both environments where significant differences were observed among control cultivars inoculated with isolate A, Coker 9733 was significantly more resistant than Coker 9766. Significant differences were observed among control cultivars inoculated with isolate B in Kinston only. In this environment, the results with isolate $\mathrm{B}$ contrasted with those observed for the natural infection treatment and the isolate A treatment; Coker 9766 was significantly more resistant than Coker 9733 and Caldwell. When the control cultivars were inoculated with a combination of isolates A plus B, Coker 9733 was more resistant than Coker 9766 in Laurel Springs 1998, more susceptible in Kinston, and not significantly different in Laurel Springs 1997.

Although significant genotype-byenvironment interactions and nonsignificant rank correlations between environments underscored the importance of multienvironment testing for SNB resistance, it did not preclude the identification of superior genotypes within any one inoculation treatment-environment combination that also displayed resistance across inoculation treatments and environments. For example, Scharen and Eyal (42) reported three distinct response classes among genotypes evaluated for SNB resistance; i.e., those with a uniformly resistant response, those with a uniformly susceptible response, and those varying in magnitude of response to isolates. The susceptible control, Coker 9766, served as the benchmark for selection of the most resistant genotypes in each of the 10 inoculation treatment-environment combinations where significant differences were observed among the 147 genotypes (Table 6). The number of genotypes with disease intensity ratings less than one LSD below Coker 9766 in the natural infection treatment varied from 21 at Laurel Springs 1997 to 132 at Kinston. The number varied in the selected isolate treatments from 85 for inoculation with isolate $\mathrm{A}$ alone at Kinston to 8 at the same location inoculated with isolate B alone.

Disease intensity in the natural infection treatment averaged over the three environments represented the best estimate of resistance that would be obtained in a conventional evaluation program without supplemental inoculation with selected isolates. The ability of supplemental inoculation versus natural infection to identify resistant genotypes within a single environment was compared using the mean disease rankings of genotypes in the natural infection treatment averaged over the three environments (Table 6). The natural infection treatment had an inherent advantage given that rankings in any one environment are a component of the mean overall environments. Nevertheless, it was an informative comparison in cases where the selected isolate treatments performed equally to the natural infection treatment. Of the 21 genotypes significantly more resistant than Coker 9766 in the natural infection treatment at Laurel Springs 1997, 12 were also found among the $38(25 \%)$ most resistant genotypes in the natural infection treatment averaged over the three environments. At this location, both inoculation with isolate $\mathrm{A}$ alone and isolate $\mathrm{B}$ alone identified as many of the top $25 \%$ of genotypes in the natural infection treatment averaged over the three environments as did the natural infection treatment per se. This result was expected given the relatively high rank correlations between treatments in this environment (Table 4),

Table 6. Correspondence between the wheat genotypes selected in 10 inoculation treatment-environment combinations and the $38(25 \%)$ most resistant genotypes averaged over the natural infection treatment (NI) in all three environments

\begin{tabular}{|c|c|c|c|c|}
\hline & \multicolumn{4}{|c|}{ No. genotypes } \\
\hline & Isolate A & Isolate B & Isolates $\mathbf{A}+\mathbf{B}$ & NI \\
\hline \multicolumn{5}{|l|}{ Laurel Springs 1997} \\
\hline More resistant than Coker 9766 & 28 & 23 & 14 & 21 \\
\hline $\begin{array}{l}\text { and among most resistant } 25 \% \\
\text { over all NI treatments }\end{array}$ & 13 & 14 & 7 & 12 \\
\hline \multicolumn{5}{|l|}{ Laurel Springs 1998} \\
\hline More resistant than Coker 9766 & $\ldots$ & $\ldots$ & 18 & 42 \\
\hline $\begin{array}{l}\text { and among most resistant } 25 \% \\
\text { over all NI treatments }\end{array}$ & $\ldots$ & $\ldots$ & 9 & 28 \\
\hline \multicolumn{5}{|l|}{ Kinston } \\
\hline More resistant than Coker 9766 & 85 & 8 & 32 & 132 \\
\hline $\begin{array}{l}\text { and among most resistant } 25 \% \\
\text { over all NI treatments }\end{array}$ & 31 & 3 & 12 & 35 \\
\hline
\end{tabular}

Table 5. Disease intensity ratings for three control cultivars of wheat evaluated in four inoculation treatments with Stagonospora nodorum in three environments in the 1997 and 1998 seasons

\begin{tabular}{|c|c|c|c|c|c|c|c|c|c|c|c|c|}
\hline \multirow[b]{3}{*}{ Cultivar } & \multicolumn{4}{|c|}{ Laurel Springs (1997) } & \multicolumn{4}{|c|}{ Laurel Springs (1998) } & \multicolumn{4}{|c|}{ Kinston (1889) } \\
\hline & \multicolumn{3}{|c|}{ Isolate } & \multirow[b]{2}{*}{ NI } & \multicolumn{3}{|c|}{ Isolate } & \multirow[b]{2}{*}{ NI } & \multicolumn{3}{|c|}{ Isolate } & \multirow[b]{2}{*}{ NI } \\
\hline & $\mathbf{A}$ & B & $\mathbf{A}+\mathbf{B}$ & & $\mathbf{A}$ & B & $A+B$ & & A & B & $A+B$ & \\
\hline Coker 9733 & $79^{z}$ & 81 & 77 & 79 & 54 & 36 & 40 & 43 & 47 & 52 & 58 & 42 \\
\hline Caldwell & 87 & 85 & 83 & 81 & 55 & 43 & 52 & 48 & 43 & 45 & 44 & 38 \\
\hline Coker 9766 & 83 & 85 & 79 & 78 & 45 & 45 & 52 & 61 & 55 & 38 & 42 & 53 \\
\hline $\operatorname{LSD}(0.05)$ & 3 & 4 & 4 & ns & ns & ns & 7 & 6 & 4 & 4 & 4 & 5 \\
\hline
\end{tabular}

${ }^{\mathrm{z}}$ Disease intensity was recorded using the Saari-Prescott double-digit scale (00 to 99$)$ where the first digit indicated the relative height of infection $(5=$ midplant and $9=$ head) and the second digit indicated the severity of disease on the highest infected leaf or the head on a scale of 0 to 9. 
which likely resulted from the primary influence of natural inoculum on epidemic development.

In Kinston, the natural infection treatment and inoculation with isolate A alone identified 82 and $92 \%$, respectively, of the top $25 \%$ most resistant genotypes in the natural infection treatment averaged over the three environments. In contrast, the treatments inoculated with isolate $\mathrm{B}$ alone and the combination of isolates $\mathrm{A}$ and $\mathrm{B}$ identified 8 and $32 \%$, respectively, of the $25 \%$ most resistant genotypes. In Laurel Springs 1998, the treatment with a combination of isolates $\mathrm{A}$ and $\mathrm{B}$ identified nine of the 28 most resistant genotypes identified by the natural infection treatment.

Our results indicated that estimates of resistance to SNB in epidemics supplemented with selected isolates did not consistently agree with estimates of host resistance in epidemics produced by natural inoculum alone. This was observed when selected isolates were used in supplemented inoculations either alone or in combination. Five of nine correlations between ratings based on inoculation with selected isolates and natural infection were significant, but three of these occurred in a single environment when the influence of selected isolates on epidemic development was likely minimal (Table 4). Natural infection resulted in high levels of SNB in all three environments and provided the opportunity to evaluate the impact of routine inoculation with selected isolates in environments where, in retrospect, supplemental inoculation may not have been necessary. This represented a practical scenario, however, because prediction of the role of natural inoculum in epidemic development is not possible at the stage of crop development when timely inoculation with selected isolates is required. While the results from Laurel Springs 1997 underscored the importance of timing of inoculation with selected isolates, the results from Laurel Springs 1998 illustrated that potential problems resulting from artificial inoculation methodologies are not confined to timing of applications. The supplemental inoculation at Laurel Springs 1998 was associated with a masking of genetic variation in two of the three selected isolate treatments. This was very detrimental to genetic progress for SNB resistance. In contrast, genetic variance for SNB resistance in the natural infection treatment at this location was two to three times that observed in any of the other inoculation treatment-environment combinations and had the third highest heritability for resistance estimates (Table 3). There were large, significant differences between the moderately resistant and susceptible control cultivars in the natural infection treatment at this location (Table 5), and identification of the most resistant genotypes was effective (Table 6). Thus, there was a clear disadvantage from using se- lected isolates versus natural infection at this location. In Laurel Springs 1997, the supplemental inoculation was not detrimental to selection effects in terms of genetic variances and heritabilities for SNB resistance (Table 3), rank correlations (Table 4), and identification of the most resistant genotypes (Table 6). In Kinston, the only significant rank correlation with the natural infection treatment was the isolate A treatment (Table 4), and there was agreement between these two treatments with respect to SNB resistance in the control cultivars (Table 5) and identification of the most resistant genotypes overall (Table 6).

Our results were more in agreement with the conclusions of McDonald et al. $(28,29)$ and Mebrate and Cooke (31), promoting the use of diverse mixtures of isolates in screening for host resistance, than with Arseniuk and Czembor (2), who suggested the use of a single aggressive isolate. Although isolate A differentiated between genotypes better than the less aggressive isolate B in the phytotron (Table 2), isolate A did not result in more disease development or greater genetic variances and heritabilities for SNB resistance in the field (Table 3). In addition, isolate A did not exhibit a clear superiority over the other two selected isolate treatments in significant rank correlations with natural inoculation (Table 4). However, it should be noted that the isolate $\mathrm{A}$ treatment was equal to the natural infection treatment at both Laurel Springs 1997 and Kinston in identification of the most resistant genotypes. This could argue for further study of selected aggressive isolates following refinement of inoculation protocols.

In summary, results did not support the routine use of supplemental inoculation of wheat breeding nurseries in North Carolina with selected isolates of $S$. nodorum, especially in conditions favorable to SNB development from natural inoculum. While one cannot rule out the feasibility of using a single aggressive isolate to ensure epidemic development, additional screening of isolates in field environments would be necessary to identify the most appropriate isolate(s). Further research on timing and inoculation methodology would be valuable also. Rather, our results indicated that priority should be given to the routine encouragement of SNB epidemics based on natural inoculum. This could include the mechanical shredding and dispersal of straw baled in infected fields the previous season, plus overhead irrigation when optimum temperatures for SNB development occurred in the spring. Such a protocol would be the most efficient for screening the 30,000 to 50,000 genotypes in a typical wheat breeding program for SNB resistance.

\section{LITERATURE CITED}

1. Allingham, E. A., and Jackson, L. F. 1981. Variation in pathogenicity, virulence, and ag- gressiveness of Septoria nodorum in Florida. Phytopathology 71:1080-1085.

2. Arseniuk, E., and Czembor, P. C. 1999. Hostparasite interactions: Stagonospora nodorum. Pages 63-70 in: Septoria and Stagonospora Diseases of Cereals: A Compilation of Global Research. M. van Ginkel, A. McNab, and J. Krupinsky, eds. CIMMYT, Mexico, D.F.

3. Arseniuk, E., Fried, P. M., Winzeler, H., and Czembor, P. 1991. Comparison of resistance of triticale, wheat and spelt to Septoria nodorum blotch and Septoria tritici blotch at the seedling and adult plant stages. Euphytica 55:43-48.

4. Bostwick, D. E., Ohm, H. W., and Shaner, G. 1993. Inheritance of Septoria glume blotch resistance in wheat. Crop Sci. 33:439-443.

5. Bronnimann, A. 1968. Investigations of Septoria nodorum Berk. of wheat. Mitt. Schweiz. Landwirtsch. 16:65-76.

6. Bronnimann, A. 1975. Contribution to the genetics of tolerance towards Septoria nodorum Berk. in wheat (Triticum aestivum L.). Z. Pflanzenzuecht. 75:138-160.

7. Cunfer, B. M., Johnson, J. W., Buntin, G. D., Barnett, R. D., and Roberts, J. J. 2001. Registration of four soft red winter wheat germplasms resistant to Stagonospora nodorum and other foliar pathogens. Crop Sci. 41:933934.

8. Cunfer, B. M., Stooksbury, D. E., and Johnson, J. W. 1988. Components of partial resistance to Leptosphaeria nodorum among seven soft red winter wheats. Euphytica 37:129-140.

9. Du, C. G., Nelson, L. R., and McDaniel, M. E 1999. Diallel analysis of gene effects conditioning resistance to Stagonospora nodorum (Berk.) in wheat. Crop Sci. 39:686-690.

10. Eyal, Z. 1999. Breeding for resistance to Septoria and Stagonosporora diseases in wheat. Pages 332-344 in: Septoria on Cereals: A Study of Pathosystems. J. A. Lucas, P. Bowyer, and H. M. Anderson, eds. CABI Publishing, New York.

11. Eyal, Z., Scharen, A. L., Prescott, J. M., and van Ginkel, M. 1987. The Septoria diseases of wheat: Concepts and methods of disease management. CIMMYT, Mexico, D.F.

12. Griffiths, E., and Ao, H. C. 1976. Dispersal of Septoria nodorum spores and spread of glume blotch of wheat in the field. Trans. Br. Mycol. Soc. 67:413-418.

13. Jeger, M. J., Jones, D. G., and Griffiths, E. 1983. Components of partial resistance of wheat seedlings to Septoria nodorum. Euphytica 32:575-584.

14. Jonsson, J. O. 1985. Evaluation of leaf resistance to Septoria nodorum in winter wheat at seedling and adult plant stage. Agri Hortique Genetica XLIII:52-68.

15. Karjalainen, R. 1985. Host-pathogen interaction between spring wheat and Septoria nodorum with reference to resistance breeding. J. Agric. Sci. Finland 57:1-66.

16. Keller, S. M., McDermott, J. M., Pettway, R. E., Wolf, M. S., and McDonald, B. A. 1997. Gene flow and sexual reproduction in the wheat glume blotch pathogen Phaeosphaeria nodorum (anamorph Stagonospora nodorum). Phytopathology 87:353-358.

17. Keller, S. M., Wolfe, M. S., McDermott, J. M., and McDonald, B. A. 1997. High genetic similarity among populations of Phaeosphaeria nodorum across wheat cultivars and regions in Switzerland. Phytopathology 87:1134-1139.

18. Koric, B. 1988. Seedling and adult stage screening for Septoria nodorum resistance in wheat. Rachis 7(1,2):31-32.

19. Krupinsky, J. M. 1997. Aggressiveness of Stagonospora nodorum isolates obtained from wheat in the northern Great Plains. Plant Dis. 81:1027-1031. 
20. Krupinsky, J. M., Schillinger, J. A., and Scharen, A. L. 1972. Resistance in wheats to Septoria nodorum. Crop Sci. 12:528-530.

21. Lancashire, P. D., and Jones, D. G. 1985. Components of partial resistance to Septoria nodorum in winter wheat. Ann. Appl. Biol. 106:541-553.

22. Large, E. C. 1954. Growth stages in cereals. Illustrations of the Feeke's scale. Plant Pathol. 3:129.

23. Leath, S., Scharen, A. L., Lund, R. E., and Dietz-Holmes, M. E. 1993. Factors associated with global occurrences of Septoria nodorum blotch and Septoria tritici blotch of wheat. Plant Dis. 77:1266-1270.

24. Littell, R. C., Nilliken, G. A., Stroup, W. W., and Wolfinger, R. D. 1996. SAS System for Mixed Models. SAS Institute, Inc., Cary, NC.

25. Luke, H. H., Barnett, R. D., and Pfahler, P. L. 1986. Development of Septoria nodorum blotch on wheat from infected and treated seed. Plant Dis. 70:252-254.

26. Manandhar, J. B., and Cunfer, B. M. 1991. An improved selective medium for the assay of Septoria nodorum from wheat seed. Phytopathology 81:771-773.

27. McDonald, B. A.. Miles, J., Nelson, L. R., and Pettway, R. E. 1994. Genetic variability in nuclear DNA in field populations of Stagonospora nodorum. Phytopathology 84:250-255.

28. McDonald, B. A., Mundt, C. C., and Zhan, J. 1999. Population genetics of Mycosphaerella graminicola and Phaeosphaeria nodorum. Pages 77-82 in: Septoria and Stagonospora Diseases of Cereals: A Compilation of Global Research. M. van Ginkel, A. McNab, and J. Krupinsky, eds. CIMMYT, Mexico, D.F.

29. McDonald, B. A., Zhan, J., Yarden, O., Hogan, K., Garton, J., and Pettway, R. E. 1999. The population genetics of Mycosphaerella graminicola and Stagonospora nodorum. Pages 44-69 in: Septoria on Cereals: A Study of Pathosystems. J. A. Lucas, P. Bowyer, and H. M. Anderson, eds. CABI Publishing, New York.
30. McIntosh, M. S. 1983. Analysis of combined experiments. Agron. J. 75:153-155.

31. Mebrate, S. A., and Cooke, B. M. 2001. Response of wheat cultivars to infection by Stagonospora nodorum isolates/mixture on detached and intact seedling leaves. Euphytica 122(2):263-268.

32. Mullaney, E. J., Martin, J. M., and Scharen, A. L. 1982. Generation mean analysis to identify and partition the components of genetic resistance to Septoria nodorum in wheat. Euphytica 31:539-545.

33. Nelson, L. R. 1980. Inheritance of resistance to Septoria nodorum in wheat. Crop Sci. 20:447-449.

34. Nelson, L. R., and Gates, C. E. 1982. Genetics of host plant resistance of wheat to Septoria nodorum. Crop Sci. 22:771-773.

35. Nelson, L. R., and Marshall, D. 1990. Breeding wheat for resistance to Septoria nodorum and Septoria tritici. Adv. Agron. 44:257-277.

36. Nyquist, W. E. 1991. Estimation of heritability and prediction of selection response in plant populations. Crit. Rev. Plant Sci. 10:235-322.

37. Rosielle, A. A., and Brown, A. G. P. 1979. Inheritance, heritability and breeding behaviour of three sources of resistance to Septoria tritici in wheat. Euphytica 28:385-392.

38. Rufty, R. C., Hebert, T. T., and Murphy, C. F. 1981. Variation in virulence of isolates of Septoria nodorum. Phytopathology 71:593596.

39. Scharen, A. L. 1964. Environmental influences on development of glume blotch in wheat. Phytopathology 54:300-303.

40. Scharen, A. L. 1999. Biology of the Septoria/Stagonospora pathogens: An overview. Pages 19-22 in: Septoria and Stagonospora Diseases of Cereals: A Compilation of Global Research. M. van Ginkel, A. McNab, and J. Krupinsky, eds. CIMMYT, Mexico, D.F.

41. Scharen, A. L., and Eyal, Z. 1980. Measurement of quantitative resistance to Septoria nodorum in wheat. Plant Dis. 64:492-496.

42. Scharen, A. L., and Eyal, Z. 1983. Analysis of symptoms on spring and winter wheat cultivars inoculated with different isolates of Septoria nodorum. Phytopathology 73:143-147.

43. Scharen, A. L., Eyal, Z., Huffman, M. D., and Prescott, J. M. 1985. The distribution and frequency of virulence genes in geographically separated populations of Leptosphaeria nodorum. Phytopathology 75:1463-1468.

44. Scharen, A. L., and Krupinsky, J. M. 1970 Cultural and inoculation studies of Septoria nodorum, cause of glume blotch of wheat Phytopathology 60:1480-1485.

45. Scott, P. R., Benedikz, P. W., and Cox, C. W. 1982. A genetic study on the relationship between height, time of ear emergence, and resistance to Septoria nodorum in wheat. Plant Pathol. 31:45-60.

46. Shah, D., Bergstrom, G. C., and Ueng, P. P. 1995. Initiation of Septoria nodorum blotch epidemics in winter wheat by seedborne Stagonospora nodorum. Phytopathology 85:452-457.

47. Shaner, G. 1999. Breeding for resistance to Septoria and Stagonospora blotches in winter wheat in the United States. Pages 127-130 in: Septoria and Stagonospora Diseases of Cereals: A Compilation of Global Research. M van Ginkel, A. McNab, and J. Krupinsky, eds. CIMMYT, Mexico, D.F.

48. Van Beuningen, L. T., and Kohli, M. M. 1990. Deviation from the regression of infection on heading and height as a measure of resistance to Septoria tritici blotch on wheat. Plant Dis. 74:488-493.

49. Wafford, J. D., and Whitbread, R. 1978. Effects of inoculation with Septoria nodorum on yield components of spring wheat. Ann. Appl. Biol. 90:323-328

50. Wilkinson, C. A., Murphy, J. P., and Rufty, R. C. 1990. Diallel analysis of components of partial resistance to Septoria nodorum in wheat. Plant Dis. 74:47-50.

51. Yan, W. 2001. GGE biplot-A Windows application for graphical analysis of multienvironment trial data and other types of two way data. Agron. J. 93:1111-1118. 